\title{
HARMONIC INVERSE SEMIGROUPS
}

\author{
by MARIO PETRICH and STUART A. RANKIN $\dagger$
}

(Received 28 July, 1988)

1. Introduction and summary. An inverse semigroup $S$ shall be said to be harmonic if every congruence on $S$ is determined by any one of its classes. In other words, if $\lambda$ and $\rho$ are congruences on $S$ having a congruence class in common, then $\lambda=\rho$. The class $\mathfrak{S}$ of all harmonic semigroups contains all bisimple inverse semigroups, as proved by Žitomirskiı [11] and also by Schein [10], and all congruence-free inverse semigroups. Moreover, $\mathfrak{S}$ is contained in the class of all 0 -simple or simple inverse semigroups, as is easy to see. We shall show that there exist non-bisimple, non-congruence-free harmonic semigroups and that there are simple inverse semigroups which are not harmonic.

The results of this paper are grouped as follows. Section 2 is a preliminary one, containing notation and a brief discussion of relevant facts.

In Section 3, we investigate the implications of two congruences having a congruence class in common. One interesting and useful observation is that two congruences having the same trace and a class in common must have an idempotent class in common. The elementary fact that any two group congruences with a class in common must be equal is essentially a consequence of this observation.

Harmonic semigroups are discussed in Section 5. The notions of kernel-harmonic and trace-harmonic semigroups are introduced and studied in Sections 6 and 7 respectively.

As mentioned earlier, simple inverse semigroups are central to these investigations. One manageable class of simple inverse semigroups consists of the Bruck extensions of inverse monoids. Section 8 contains a characterization of harmonic Bruck extensions, as well as several additional results which are illuminating in view of the various conditions studied in Sections 6 and 7. It is in Section 8 that we construct a class of harmonic semigroups which properly contains the class of all bisimple or congruence-free inverse semigroups. The characterization theorem also provides a ready source of examples of simple non-harmonic inverse semigroups, thus showing that $\mathfrak{S}$ is indeed properly contained in the class of all (0-)simple inverse semigroups. In particular, we are able to answer a question posed by Schein in [10]: it is not true that every congruence contained in $\mathscr{D}$ on a (0-)simple inverse semigroup is determined by each of its congruence classes. Theorem 3.4 and the discussion in Section 4 provide considerable information about the extent to which a congruence is determined by a given congruence class.

We conclude the paper with a brief discussion in Section 9 of $E$-unitary inverse semigroups in the context of harmonicity. The discussion follows the general theme developed by Reilly in [9].

2. Notation and relevant facts. Throughout this paper, $S$ will denote an inverse semigroup and $E$ its semilattice of idempotents. For any subset $A$ of $S$, we shall write $E_{A}$ for $E \cap A$.

$†$ This research was supported by NSERC.

Glasgow Math. J. 31 (1989) 335-351. 
We shall use $\varepsilon$ and $\omega$ to denote the equality relation and the universal relation, respectively, on any set. The reader is cautioned that occasionally a discussion will involve the equality relations (or the universal relations) on two different sets. We feel that the context is sufficient to forestall any ambiguity. We note that the symbol $\omega$ is also used to denote the closure operator, that is, for $A \subseteq S, A \omega=\{x \in S \mid x E \cap A \neq \varnothing\}$. Once again, the meaning is made clear by the context.

The kernel-trace description of congruences on an inverse semigroup plays a central role in much of this paper. For a complete discussion of kernel and trace, see [6]. For any congruence $\rho$ on $S$, the kernel of $\rho$, denoted by $\operatorname{ker} \rho$, is the union of the idempotent classes of $\rho$, while the trace of $\rho$, denoted by $\operatorname{tr} \rho$, is the restriction of $\rho$ to $E$. The trace of any congruence on $S$ is a normal congruence on $E$, that is, a congruence on $E$ which is invariant under conjugation by elements of $S$. Conversely, every normal congruence on $E$ is the trace of some congruence on $S$ and the set of all congruences on $S$ with a given trace forms an interval in the congruence lattice of $S$. For any congruence $\rho$ on $S$, the greatest congruence with trace that of $\rho$ is denoted by $\rho_{\max }$. It is conventional to let $\mu$ denote $\varepsilon_{\max }$.

Recall that for any subset $A$ of $S$, the principal congruence on $S$ induced by $A$, denoted by $P_{A}$, is defined by

$$
a P_{A} b \Leftrightarrow\left(x a y \in A \Leftrightarrow x b y \in A \text { for all } x, y \in S^{1}\right) .
$$

$P_{A}$ is the greatest congruence on $S$ which saturates $A$. Furthermore, a subset $K$ of $S$ is the kernel of some congruence on $S$ if and only if $K=\operatorname{ker} P_{K}$.

Finally, if $S$ and $T$ are semigroups and $\alpha: S \rightarrow T$ is a homomorphism, then for any relation $\gamma$ on $T$, the pullback of $\gamma$ to $S$ along $\alpha$ is the relation ${ }^{\alpha} \gamma$ on $S$ defined by

$$
x^{\alpha} \gamma y \Leftrightarrow(x \alpha) \gamma(y \alpha)
$$

3. Congruences having a class in common. We should like to know to what extent two congruences must agree if they have a congruence class in common. In this section, we establish some lower bounds for the amount of agreement between two such congruences. A particular case of importance in later sections occurs when one of the congruences is a group congruence.

Our first lemma presents the essence of Žitomirskiı̈'s theorem (see Corollary 3.6).

LeMma 3.1. For any $x \in S$, we have $\left.P_{\{x\}}\right|_{x \mathscr{D}}=\left.\varepsilon\right|_{x \mathscr{D}}$.

Proof. Let $u, v \in X \mathscr{D}$ and suppose that $u P_{\{x\}} v$. Since $u \mathscr{D} x$ we have $u u^{-1} \mathscr{D} x^{-1} x$ and so there exists $t \in S$ with $u u^{-1}=t t^{-1}, t^{-1} t=x^{-1} x$. But then $x=x t^{-1} t=x t^{-1} t t^{-1} t=$ $\left(x t^{-1}\right) u\left(u^{-1} t\right)$ and so by hypothesis we obtain $x=\left(x t^{-1}\right) v\left(u^{-1} t\right)$. Left multiplication by $x^{-1}$ produces $x^{-1} x=x^{-1} x t^{-1} v u^{-1} t=t^{-1} v u^{-1} t$ and then conjugation by $t$ yields $u u^{-1}=$ $u u^{-1} y u^{-1}$. Thus $u \leqslant v$ and by symmetry we must have $v \leqslant u$, whence $u=v$, as required.

We remark that the preceding lemma can also be obtained as a consequence of [3, Corollary 2].

In preparation for our first result, we require two additional facts. In order to avoid cumbersome notation we shall adopt the following convention. For any congruence $\rho$ on 
$S$, we shall denote Green's $\mathscr{D}$ relation on both $S$ and $S / \rho$ by $\mathscr{D}$, and the pullback of $\mathscr{D}$ on $S / \rho$ to $S$ along the canonical homomorphism of $S$ onto $S / \rho$ by ${ }^{\rho} \mathscr{D}$.

LEMMA 3.2. For any congruence $\rho$ on $S$, we have $\rho \vee \mathscr{D}={ }^{\rho} \mathscr{D}$, where the join is taken in the lattice of equivalence relations on $S$.

Proof. It is evident that $\rho \vee \mathscr{D} \subseteq{ }^{\rho} \mathscr{D}$. Conversely, suppose that $x, y \in S$ are such that $(x \rho) \mathscr{D}(y \rho)$ in $S / \rho$. By [2, Lemma 9], there exist $a, b \in S$ with $a \mathscr{D} b$ and $x \rho a, y \rho b$. Thus $x \rho \mathscr{D} \rho y$ and so $\rho_{\mathscr{D}} \subseteq \rho \mathscr{D} \rho \subseteq \rho \vee \mathscr{D}$.

LeMma 3.3. Let $S$ and $T$ be semigroups, $\gamma: S \rightarrow T$ a surjective homomorphism, and $H \subseteq S$ a subset saturated by $\gamma$. Then the following hold.

(i) $P_{H}={ }^{\gamma}\left(P_{H \gamma}\right)$.

(ii) For any congruence $\rho$ on $T,\left.\left({ }^{\gamma} \rho\right)\right|_{H}={ }^{\gamma}\left(\left.\rho\right|_{H \gamma}\right)$.

Proof. (i) Since $P_{H \gamma}$ saturates $H \gamma$ and $\gamma$ saturates $H$, it follows that ${ }^{\gamma}\left(P_{H \gamma}\right)$ saturates $H$, whence ${ }^{\gamma}\left(P_{H \gamma}\right) \subseteq P_{H}$. Conversely, suppose that $u P_{H} v$ for $u, v \in S$. The surjectivity of $\gamma$ then implies that $(u \gamma) P_{H \gamma}(v \gamma)$ and so $P_{H} \subseteq{ }^{\gamma}\left(P_{H \gamma}\right)$.

(ii) Let $x, y \in H$ and suppose that $(x \gamma) \rho(y \gamma)$. Then $\left.(x \gamma) \rho\right|_{H \gamma}(y \gamma)$ and so $x^{\gamma}\left(\left.\rho\right|_{H \gamma}\right) y$. Conversely, if $x, y \in S$ are such that $x \gamma, y \gamma \in H \gamma$ and $(x \gamma) \rho(y \gamma)$, then $x, y \in H$ and $x^{\gamma} \rho y$ whence $\left.x\left({ }^{\gamma} \rho\right)\right|_{H} y$.

THEOREM 3.4. Let $\rho$ be a congruence on $S$. Then we have $\left.\rho\right|_{x(\rho \vee \mathscr{D})}=\left.P_{x \rho}\right|_{x(\rho \vee \mathscr{D})}$ for any $x \in S$.

Proof. Apply Lemma 3.3(i) to $H=x \rho$ and $\gamma=\rho^{\natural}: S \rightarrow S / \rho$ to obtain $P_{x \rho}=\gamma\left(P_{\{x \rho\}}\right)$. Then by Lemma 3.3(ii) we have $\left.P_{x \rho}\right|_{x(\rho \vee \mathscr{D})}={ }^{\gamma}\left(\left.P_{\{x \rho\}}\right|_{(x(\rho \vee \mathscr{D})) \gamma}\right)$. But by Lemma 3.2, we have $\rho \vee \mathscr{D}={ }^{\rho} \mathscr{D}$ and so $(x(\rho \vee \mathscr{D})) \gamma=(x \rho) \mathscr{D}$. Observe as well that by Lemma 3.1 we obtain $\left.P_{\{x \rho\}}\right|_{(x \rho) \mathscr{D}}=\left.\varepsilon\right|_{(x \rho) \mathscr{D}}$. These observations, together with one additional application of Lemma 3.3(ii), yield

$$
\left.\left.P_{x \rho(\rho \vee \mathscr{D})}\right|^{\gamma} P_{\{x \rho\}}\right|_{(x(\rho \vee \mathscr{D})) \gamma}={ }^{\gamma}\left(\left.P_{\{x \rho\}}\right|_{(x \rho) \mathscr{D}}\right)={ }^{\gamma}\left(\left.\varepsilon\right|_{(x \rho) \mathscr{D}}\right)=\left.{ }^{\gamma}(\varepsilon)\right|_{x(\rho \vee \mathscr{D})}
$$

and since $\rho={ }^{\gamma} \varepsilon$, the result follows.

Corollary 3.5. Let $\lambda$ and $\rho$ be congruences on $S$ and $c \in S$ with $c \lambda=c \rho$. Then $\left.\lambda\right|_{A}=\left.\rho\right|_{A}$ for $A=c((\lambda \vee \mathscr{D}) \cap(\rho \vee \mathscr{D}))$.

We remark that the proposition in [10] is a special case of Corollary 3.5.

COROLlARY 3.6. $([10,11])$. Every congruence on a $(0-)$ bisimple inverse semigroup is uniquely determined by any one of its (non-zero) classes.

Proof. Let $\rho \neq \omega$ be a congruence on $S$ and let $c \in S \backslash\{0\}$. Then $c(\rho \vee \mathscr{D})=S \backslash\{0\}$ and so $\rho=P_{c \rho}$.

We remark that Corollary 3.6 uses the fact that $\left.(\rho \vee \mathscr{D})\right|_{S \backslash\{0\}}=\left.\omega\right|_{s \backslash\{0\}}$, which follows from $\left.\mathscr{D}\right|_{S \backslash\{0\}}=\left.\omega\right|_{S \backslash\{0\}}$ for (0)-bisimple inverse semigroups. This allows us to conclude that every congruence on $S$ is the principal congruence on any of its (non-zero) classes. 
It is evident that we could revise Corollary 3.6 to apply to a restricted class of congruences on an arbitrary inverse semigroup if the class has the property that the join of any of its members with $\mathscr{D}$ is universal on $S$ or on $S \backslash\{0\}$. We shall make use of the next result in this context. For any congruence $\rho$ on an inverse semigroup $S, \varepsilon \cup \operatorname{tr} \rho$ is an equivalence relation on $S$. We shall denote $(\varepsilon \cup \operatorname{tr} \rho) \vee \mathscr{D}$ simply by $\operatorname{tr} \rho \vee \mathscr{D}$.

Lemma 3.7. For any congruence $\rho$ on $S, \rho \vee \mathscr{D}=\operatorname{tr} \rho \vee \mathscr{D}$.

Proof. Since $\varepsilon \cup \operatorname{tr} \rho \subseteq \rho$, we have $\operatorname{tr} \rho \vee \mathscr{D} \subseteq \rho \vee \mathscr{D}$. Conversely, if $x(\rho \vee \mathscr{D}) y$ for some $x, y \in S$, then $(x \rho) \mathscr{D}(y \rho)$ by Lemma 3.2. This implies that $x x^{-1} \rho a a^{-1} \mathscr{D} a^{-1} a \rho y y^{-1}$ for some $a \in S$. But then $x \mathscr{D} x x^{-1}(\operatorname{tr} \rho \vee \mathscr{D}) y y^{-1} \mathscr{D} y$ and so $x(\operatorname{tr} \rho \vee \mathscr{D}) y$, as required.

COROLlaRY 3.8. Every group congruence on an inverse semigroup $S$ is completely determined by any of its congruence classes. Specifically, if $\rho$ is a group congruence on $S$, then $\rho=P_{a \rho}$ for all $a \in S$.

Proof. By Lemma 3.7 we have $\rho \vee \mathscr{D}=\omega$ for a group congruence $\rho$. The result follows now by Theorem 3.4.

The next corollary presents an interesting generalization of the preceding situation and will be used in the sequel.

COROllary 3.9. Let $\lambda$ and $\rho$ be congruences on an inverse semigroup $S$ with $\operatorname{tr} \lambda=\operatorname{tr} \rho$. If $\lambda$ and $\rho$ have a congruence class in common then they have an idempotent congruence class in common. More precisely, if $a \lambda=a \rho$ for some $a \in S$, then $\left(a a^{-1}\right) \lambda=\left(a a^{-1}\right) \rho$.

Proof. By Theorem 3.4, we have $\left.\lambda\right|_{a(\lambda \vee \mathscr{D})}=\left.P_{a \lambda}\right|_{a(\lambda \vee \mathscr{D})}$ and $\left.\rho\right|_{a(\rho \vee \mathscr{D})}=\left.P_{a \rho}\right|_{a(\rho \vee \mathscr{D})}$. Now by Lemma 3.7 we obtain $a(\lambda \vee \mathscr{D})=a(\operatorname{tr} \lambda \vee \mathscr{D})=a(\operatorname{tr} \rho \vee \mathscr{D})=a(\rho \vee \mathscr{D})=A$, say. Finally, since both $\lambda$ and $\rho$ saturate $A$ and since $a a^{-1} \in A$, we obtain $\left(a a^{-1}\right) \lambda=\left(a a^{-1}\right) \rho$.

It is interesting to observe that if trace is replaced by kernel in the preceding corollary, the resulting statement is false. We are grateful to the referee for the following example. Let $I$ be the direct product of the two element chain and $Z_{2}$ and let $S$ be $I$ with 1 adjoined. Let $\pi=\sigma \cap \rho_{I}$ where $\sigma$ denotes the least group congruence on $S$ and $\rho_{I}$ denotes the Rees congruence. Then $\sigma$ and $\pi$ are congruences each with kernel $E$ and for which the two nonidempotent elements form a common congruence class, yet $\sigma$ and $\pi$ do not have an idempotent class in common.

LEMMA 3.10. Let $\rho$ be a group congruence and $\lambda$ a congruence on $S$. If a $\rho=a \lambda$ for some $a \in S$ then $E_{a a^{-1}} \subseteq\left(a a^{-1}\right) \lambda$.

Proof. Since $\operatorname{tr} \rho=\omega$ we have $E a a^{-1} \subseteq\left(a a^{-1}\right) \rho a a^{-1} \subseteq(a \rho) a^{-1}=(a \lambda) a^{-1} \subseteq\left(a a^{-1}\right) \lambda$.

The following proposition and its corollary will be required in later sections.

Proposition 3.11. Let $\rho$ be a group congruence and $\lambda$ an idempotent separating congruence on $S$. If $a \rho=a \lambda$ for some $a \in S$ then $a \mathscr{D}$ is a group ideal of $S$. 
Proof. Since $\lambda \subseteq \mathscr{D}$, we have by Corollary 3.5 that $\left.\lambda\right|_{a \mathscr{D}}=\left.\rho\right|_{a \mathscr{D}}$ whence $a \mathscr{D}$ contains a unique idempotent. Thus $a \mathscr{D}$ is a group. Now by Corollary 3.9 and Lemma 3.10, we have $E a a^{-1} \subseteq E \cap\left(a a^{-1}\right) \lambda=\left\{a a^{-1}\right\}$. Thus the identity of $a \mathscr{D}$ is a zero for $E$, from which it follows that $a \mathscr{D}$ is an ideal of $S$.

COROLlaRY 3.12. If $S$ is a simple inverse semigroup, no group congruence on $S$ can have a congruence class in common with a non-group congruence on $S$.

Proof. Let $\rho$ be a group congruence on $S$ and $\lambda$ a non-group congruence on $S$. Suppose that $a \lambda=a \rho$ for some $a \in S$. Then for $\delta=\lambda \cap \rho, a \delta=a \rho$ and $\delta \subseteq \rho$. On $S / \delta$, which is not a group, we have the group congruence $\rho^{\prime}=\rho / \delta$. Furthermore, $(a \delta) \rho^{\prime}=$ $(a \delta) \varepsilon$ and so by Proposition 3.11, we see that $(a \delta) \mathscr{D}$ is a group ideal of $S / \delta$, hence a proper ideal of $S / \delta$. Thus $S$ has a proper ideal. This establishes the contrapositive of the statement of the corollary.

4. Congruences with a common $\mathscr{D}$-section. We are now in a position to present necessary and sufficient conditions on two congruences in order that they should be equal.

Definition 4.1. Let $\rho$ be a congruence on an inverse semigroup $S$. A $\mathscr{D}$-section for $\rho$ is a set $\Delta$ of congruence classes of $\rho$ with the property that each $\mathscr{D}$-class of $S$ intersects at least one member of $\Delta$.

Proposition 4.2. Two congruences $\lambda$ and $\rho$ on an inverse semigroup $S$ are equal if and only if they have a common $\mathscr{D}$-section.

Proof. Since $\{a \rho \mid a \in S\}$ is a $\mathscr{D}$-section for $\rho$, it follows that $\lambda=\rho$ implies $\lambda$ and $\rho$ have a common $\mathscr{D}$-section. Suppose now that $\Delta$ is a $\mathscr{D}$-section for $\lambda$ such that $a \lambda \in \Delta$ implies $a \lambda=a \rho$. We show that $\lambda=\rho$. Let $x \in S$. Then for some $a \mathscr{D} x, a \lambda \in \Delta$. But then by Theorem 3.4 we have $x \lambda=x P_{a \lambda}=x P_{a \rho}=x \rho$. Thus $\lambda=\rho$.

Corollary 4.3. Let $\rho$ be a congruence on an inverse semigroup $S$ and let $A$ be $a$ cross-section of $\mathscr{D}$. Then $\rho=\bigcap_{a \in A} P_{a \rho}$.

Proof. In view of Proposition 4.2, it suffices to prove that $x \rho=x\left(\bigcap_{a \in A} P_{a \rho}\right)$ for each $x \in A$. Since $\rho \subseteq \bigcap_{a \in A} P_{a \rho}$, we get $x \rho \subseteq x\left(\bigcap_{a \in A} P_{a \rho}\right)$. Now if $b \in x\left(\bigcap_{a \in A} P_{a \rho}\right)$, we have in particular that $b \in x P_{x \rho}=x \rho$ and so $x\left(\bigcap_{a \in A} P_{a \rho}\right) \subseteq x \rho$.

We close this section with an interesting variation of the preceding result (see [7] for a related discussion of transitive representatives of an inverse semigroup).

LeMmA 4.4. Let $\rho$ be a congruence on $S$ and $a, b \in S$. If $a \mathscr{D b}$ then $P_{(a \rho) \omega}=P_{(b \rho) \omega}$.

Proof. There exists $u \in S$ such that $a a^{-1}=u u^{-1}$ and $b^{-1} b=u^{-1} u$. Assume that $c P_{(a \rho) \omega} d$ and let $x, y \in S^{1}$ be such that $x c y \in(b \rho) \omega$. Then $b b^{-1} x c y \rho b$, from which we 
obtain $b^{-1} x c y \rho b^{-1} b=u^{-1} u$, which implies that $u b^{-1} x c y u^{-1} \rho u u^{-1}=a a^{-1}$. It follows that $\left(u b^{-1} x\right) c\left(y u^{-1} a\right) \rho a$ and the hypothesis implies that $\left(u b^{-1} x\right) d\left(y u^{-1} a\right) a^{-1} a \rho a$. Hence

$$
u^{-1} u b^{-1} x d y u^{-1} a a^{-1} u \rho u^{-1} a a^{-1} u=b^{-1} b
$$

and so $b b^{-1}(x d y) b^{-1} b \rho b$. Thus $x d y \in(b \rho) \omega$. By symmetry, we conclude that $c P_{(b \rho) \omega} d$ and so $P_{(a \rho) \omega} \subseteq P_{(b \rho) \omega}$. By symmetry again, we obtain the equality.

Proposition 4.5. Let $\rho$ be a congruence on $S$ and $A$ a cross-section of $\mathscr{D}$. Then $\rho=\bigcap_{a \in A} P_{(a \rho) \omega}$.

Proof. By Lemma 4.4, it suffices to show that $\rho=\bigcap_{a \in A} P_{(a \rho) \omega}$. Now the inclusion $\rho \subseteq \bigcap_{a \in A} P_{(a \rho) \omega}$ follows from maximality of $P_{(a \rho) \omega}$ and the fact that $(a \rho) \omega$ is saturated by $\rho$. Assume that $c\left(\bigcap_{a \in A} P_{(a \rho) \omega}\right) d$ so that $x c y a^{-1} a \rho a$ if and only if $x d y a^{-1} a \rho a$ for all $x, y \in S^{1}$. From $x=y=1$ and $c=a$ we get $d a^{-1} a \rho c$. Symmetrically, we have $c a^{-1} a \rho d$ and thus $c \rho d a^{-1} a \rho c a^{-1} a \rho d$, as required.

5. Harmonic inverse semigroups. It is natural enough, given Žitomirskiī's theorem (see Corollary 3.6), to wonder whether or not the collection of all inverse semigroups with the property that every congruence is determined by any of its (non-zero) classes can be described in a useful way. This question was raised by Schein in [10] and provides the motivation for the investigation described in this and subsequent sections. The term (0-)tight was introduced in [10] to refer to semigroups for which every congruence is determined by each of its (non-zero) congruence classes.

Definition 5.1. An inverse semigroup $S$ shall be said to be harmonic if every congruence on $S$ is determined by any of its classes.

First note that the above phrase is equivalent to the assertion that if $\lambda$ and $\rho$ are congruences on $S$ and $a \lambda=a \rho$ for some $a \in S$, then $\lambda=\rho$. This is the form in which the property of harmonicity will be used. Even in this form, the property is not easy to visualize except as a generalization of the well-known fact that it holds in stronger structures than semigroups, such as groups or rings. The next simple characterization provides the first illumination of harmonicity in different terms.

Lemma 5.2. An inverse semigroup $S$ is harmonic if and only if $\rho=P_{a \rho}$ for every congruence $\rho$ on $S$ and every $a \in S$.

Proof. Necessity. Since $a \rho$ is a congruence class, it follows that $a \rho$ is a $P_{a \rho}$-class. The hypothesis then implies that $\rho=P_{a \rho}$.

Sufficiency. Let $\lambda$ and $\rho$ be congruences on $S$ and assume that $a \lambda=a \rho$ for some $a \in S$. Using the hypothesis, we obtain $\lambda=P_{a \lambda}=P_{a \rho}=\rho$, as required.

From the above characterizations there naturally emerges a property weaker than harmonicity which will play an important role in the sequel. First recall that a subset $A$ of a semigroup $S$ is disjunctive if $P_{A}=\varepsilon$. 
Definition 5.3. A semigroup $S$ has $s d$-property if each one-element subset of $S$ is disjunctive.

Thus the sd-property says that "singletons are disjunctive". In comparison with Lemma 5.2, the sd-property imposes the condition $\rho=P_{a \rho}$ for all $a \in S$ only for $\rho=\varepsilon$.

We will be interested in classes of harmonic semigroups as well as classes of semigroups each of which has sd-property. It will thus be convenient to extend the above concepts as follows.

Definition 5.4. A class $\mathfrak{F}$ of inverse semigroups is said to be:

(i) an $s d$-class if every semigroup in $\mathfrak{F}$ has sd-property;

(ii) $h$-closed if it is closed under homomorphic image;

(iii) harmonic if every semigroup in $\mathfrak{F}$ is harmonic.

Denote by $\mathfrak{S}$ the class of harmonic semigroups.

LEMMA 5.5. Let $S$ and $T$ be semigroups and $\alpha: S \rightarrow T$ a surjective homomorphism.

(i) For $\lambda, \rho \in \mathscr{C}(T),{ }^{\alpha} \lambda={ }^{\alpha} \rho$ if and only if $\lambda=\rho$.

(ii) For $H \subseteq S$ with $H \alpha \alpha^{-1}=H$, we have ${ }^{\alpha}\left(P_{H \alpha}\right)=P_{H}$.

Proof. (i) Obviously, if $\lambda=\rho$ then ${ }^{\alpha} \lambda={ }^{\alpha} \rho$. Suppose now that ${ }^{\alpha} \lambda={ }^{\alpha} \rho$. For $x, y \in T$ let $x^{\prime}, y^{\prime} \in S$ be such that $x^{\prime} \alpha=x, y^{\prime} \alpha=y$. Then

$$
x \lambda y \Leftrightarrow x^{\prime \alpha} \lambda y^{\prime} \Leftrightarrow x^{\prime \alpha} \rho y^{\prime} \Leftrightarrow x \rho y
$$

and so $\lambda=\rho$.

(ii) Extend $\alpha: S^{1} \rightarrow T^{1}$ by $1 \alpha=1$. Let $x, y \in S$. For any $u, v \in S^{1}$, we have $u x v \in H \Leftrightarrow$ $(u \alpha)(x \alpha)(v \alpha) \in H \alpha$ since $H \alpha \alpha^{-1}=H$. Now the surjectivity of $\alpha$ implies that for all $u, v \in S^{1}, u x v \in H \Leftrightarrow u y v \in H$ if and only if for all $u, v \in T^{1}, u(x \alpha) v \in H \alpha \Leftrightarrow u(y \alpha) v \in$ $H \alpha$. Thus $P_{H}={ }^{\alpha}\left(P_{H \alpha}\right)$.

The stage is now set for the completion of the first part of our program, namely a characterization of the class $\mathfrak{G}$.

THEOREM 5.6. $\mathfrak{S}$ is the greatest $h$-closed sd-class of inverse semigroups.

Proof. First, we show that $\mathfrak{S}$ is such a class. It is apparent from Lemma 5.2 that $\mathfrak{W}$ is an sd-class. Let $S \in \mathfrak{S}$ and let $\alpha: S \rightarrow T$ be a surjective homomorphism. Suppose that $\lambda, \rho \in \mathscr{C}(T)$ are such that $a \lambda=a \rho$ for some $a \in T$. For $a^{\prime} \in S$ with $a^{\prime} \alpha=a$, we have $a^{\prime}\left({ }^{\alpha} \lambda\right)=a^{\prime}\left({ }^{\alpha} \rho\right)$ whence ${ }^{\alpha} \lambda={ }^{\alpha} \rho$ by hypothesis and then $\lambda=\rho$ by Lemma $5.5(\mathrm{i})$. Thus $T \in \mathfrak{B}$ and so $\mathfrak{S}$ is h-closed.

Now let $\mathfrak{F}$ be an h-closed sd-class of inverse semigroups. Let $S \in \mathfrak{F}$ and $\rho \in \mathscr{C}(S)$. Then for any $a \in S, a \rho$ is saturated by the canonical homomorphism $\pi=\rho^{\natural}: S \rightarrow S / \rho$. By Lemma 5.5(ii), we have $P_{a \rho}={ }^{\pi}\left(P_{(a \rho) \pi}\right)={ }^{\pi}\left(P_{\{a \rho\}}\right)$. By hypothesis, $S / \rho \in \mathfrak{F}$ and thus every singleton of $S / \rho$ is disjunctive. This yields $P_{a \rho}={ }^{\pi} \varepsilon=\rho$ and so $S \in \mathfrak{S}$.

As for whether of not $\mathfrak{S}$ is closed under finite direct products or division, we observe that the direct product of two congruence-free inverse semigroups with zero has a proper non-zero ideal and it follows from the next proposition that such a semigroup is not 
harmonic. It is clear that the class $\mathfrak{B}$ is not even closed under taking of inverse subsemigroups.

The preceding theorem will come in quite handy in recognizing whether a given class of inverse semigroups is harmonic; indeed it suffices to verify whether it has sd-property and is h-closed, which in some cases may be easier to establish.

The second part of our program is to establish some easily recognizable bounds for the class $\mathfrak{S}$. We turn our attention now to this problem.

Proposition 5.7. Every bisimple or congruence-free inverse semigroup is harmonic. If an inverse semigroup $S$ is harmonic, then $S$ is either simple or else $S$ is 0-simple and congruence-free.

Proof. The class of bisimple inverse semigroups is h-closed and by Lemma 3.1 it is an sd-class. Now apply Theorem 5.6. (Alternatively, one could use Corollary 3.6 directly to establish that every bisimple inverse semigroup is harmonic.) That a congruence-free semigroup is harmonic is immediate.

Next, let $S$ be a harmonic semigroup. Assume that $S$ has a proper ideal $I$. Then the Rees congruence $\rho_{I}$ has the property that $a \rho_{I}=a \varepsilon$ for any $a \in S \backslash I$. The hypothesis then yields $\rho_{I}=\varepsilon$ so that $I$ is a singleton. Consequently $S$ is 0 -simple. In a 0 -simple semigroup every non-universal congruence $\rho$ satisfies $0 \rho=\{0\}=0 \varepsilon$ and so by hypothesis, $\rho=\varepsilon$. Thus $S$ is congruence-free in this case.

In view of the last part of the above proposition, we shall restrict our attention to harmonic simple semigroups.

Note that Lemma 5.2 implies that in a harmonic semigroup all congruences are principal. That this property is strictly weaker than harmonicity is exhibited by the example of the symmetric inverse semigroup on a finite set. For this semigroup, every congruence is principal [7, Proposition 6.7], yet according to Proposition 5.7, it is not harmonic except in the trivial case when $S$ has only one element.

The third part of our program here consists of finding new classes of harmonic semigroups. We have seen that the class of bisimple inverse semigroups is an h-closed sd-class and, of course, the class of congruence-free inverse semigroups is such a class. Thus the class of all congruence-free or bisimple semigroups is an h-closed sd-class. To the best of our knowledge, this class contains all previously known examples of harmonic semigroups. We will construct an h-closed sd-class of inverse semigroups which properly contains the class of all inverse semigroups which are either congruence-free or bisimple. The basic tool in our study will be the Bruck extension; the entire discussion comprises Section 8. In the next two sections we consider some variants of the notion of harmonicity.

6. Kernel-harmonic inverse semigroups. Corollary 3.8 can be formulated as a property of the variety $\mathscr{G}$ of groups. This leads to our next proposition.

Proposition 6.1. Let $\mathscr{V}$ be a variety of inverse semigroups. Then $\mathscr{V} \subseteq \mathscr{G}$ if and only if it has the following property: (*) if $S$ is an inverse semigroup, $\lambda, \rho \in \mathscr{C}(S), S / \lambda, S / \rho \in \mathscr{V}$, $a \lambda=a \rho$ for some $a \in S$, then $\lambda=\rho$. 
Proof. By Corollary 3.8, property $\left({ }^{*}\right)$ holds for $\mathscr{V}=\mathscr{G}$ and hence it holds for any subvariety $\mathscr{V}$ of $\mathscr{G}$.

Assume that $\mathscr{V} \nsubseteq \mathscr{G}$. Then $\mathscr{S} \subseteq \mathscr{V}$, where $\mathscr{S}$ denotes the variety of all semilattices. Letting $Y=\{a, b, 0\}$ where $a b=b a=0, a^{2}=a, b^{2}=b$ and 0 acts as the zero, we obtain a semilattice. Then $\{a\},\{0, b\}$ is a partition defining a congruence $\rho$ on $S$ such that $a \rho=a \varepsilon$ with $Y / \rho, Y / \varepsilon \in \mathscr{V}$ and $\rho \neq \varepsilon$. Hence (*) fails. The converse assertion of the proposition now follows by contrapositive.

Motivated by the restrictions implied by the above result, we contemplate briefly a property which, we note, is weaker than harmonicity:

$$
a \lambda=a \rho \text { for some } a \in S \Rightarrow \lambda \vee \sigma=\rho \vee \sigma .
$$

The observation that $\operatorname{ker}(\lambda \vee \sigma)=(\operatorname{ker} \lambda) \omega$ then leads us to the property to be considered in this section.

Definition 6.2. An inverse semigroup $S$ has property $\mathbf{H}^{\mathrm{ker}}$ or is said to be kernel-harmonic if any two congruences which have a class in common have the same kernel.

An obvious modification of the proof of Lemma 5.2 establishes

Lemma 6.3. An inverse semigroup $S$ is kernel-harmonic if and only if for every congruence $\rho$ on $S$ and every $a \in S$, we have $\operatorname{ker} \rho=\operatorname{ker} P_{a \rho}$.

We now present a general classification of inverse semigroups with property $\mathbf{H}^{\mathrm{ker}}$.

THEOREM 6.4. An inverse semigroup $S$ is kernel-harmonic if and only if it is of one of the following types:

(i) a semilattice,

(ii) a simple kernel-harmonic semigroup

(iii) a 0-simple semigroup all of whose non-universal congruences are idempotent pure.

Proof. Direct part. Assume that $S$ has property $\mathbf{H}^{\mathrm{ker}}$ but is neither a semilattice nor simple. Let $I$ be a proper ideal of $S$. Then $\rho_{I}$ and $\varepsilon$ have a class $\{a\}$ in common, where $a \in S \backslash I$. Hence $\operatorname{ker} \rho_{I}=\operatorname{ker} \varepsilon$ so that $I \cup E=E$. But then $I \subseteq E$, that is, every proper ideal of $S$ is a semilattice. Next, let $a \in S \backslash E$. Then $a \in J(a)$ and hence $J(a)=S$. If $I(a)=\varnothing$, then $J(a)$ is simple, so $S$ is simple, a contradiction. Thus $I(a) \neq \varnothing$ and is a semilattice by the above. It follows that $S$ is an ideal extension of the semilattice $Y=I(a)$ by the 0 -simple semigroup $T=J(a) / I(a)$. Furthermore, by assumption, $T$ is not a semilattice. Now, since $J(a)=S$, it follows that for any $t \in S \backslash Y, S t S=S$. In particular, we see that for any $e \in Y$ and any $f \in E \backslash Y$, we have $e \in S f S$, say $e=r f s$ for some $r, s \in S$. But then $e=(e r) f(s e) \leqslant f$ since $e r$, se $\in \subseteq E$. As a result, for any $t \in S \backslash Y$ and any $e \in Y$, we have $t e=(t e)^{-1} t e=e\left(t^{-1} t\right)=e$ since $t^{-1} t \in E \backslash Y$ implies that $e \leqslant t^{-1} t$. Likewise, $e t=e$. Observe now that if there exist $s, t \in S \backslash Y$ with $s t=e \in Y$, then for all $f \in Y, f=t f=$ $s(f)=e f$, whence $f \leq e$. Thus either $S \backslash Y$ is without zero-divisors, or else $Y$ has an identity, $e$ say, and $s t=e$ for all $s, t \in S \backslash Y$ for which $s t \in Y$. In the former case, the 
equivalence relation $\rho$ having $S \backslash Y$ and singletons as classes is a congruence on $S$ while in the latter case, the equivalence relation $\rho$ whose classes are $(S \backslash Y) \cup\{e\}$ and singletons is a congruence on $S$. In either case, if $|Y|>1$, then $\rho$ and $\varepsilon$ have a class in common and so by property $\mathbf{H}^{\mathrm{ker}}$ we obtain $S=\operatorname{ker} \rho=\operatorname{ker} \varepsilon=E$, a contradiction. Thus $|Y|=1$. We have therefore established that $S$ is a 0 -simple inverse semigroup. But then any proper congruence $\rho$ has $\{0\}$ as a class, whence by property $\mathbf{H}^{\mathrm{ker}}$ we have $\operatorname{ker} \rho=\operatorname{ker} \varepsilon=E$ and so all proper congruences are idempotent pure.

Converse. Property $\mathbf{H}^{\mathrm{ker}}$ is trivially satisfied in semilattices. It thus remains to consider semigroups of type (iii). Let $\lambda$ and $\rho$ be congruences on $S$ such that $a \lambda=a \rho$ for some $a$. If $a \lambda=S$, both $\lambda$ and $\rho$ are universal so $\operatorname{ker} \lambda=\operatorname{ker} \rho=S$. Otherwise, $a \lambda \neq S$ so that neither $\lambda$ nor $\rho$ is universal and hence, by hypothesis, $\operatorname{ker} \lambda=\operatorname{ker} \rho$. Therefore $S$ satisfies property $\mathbf{H}^{\mathrm{ker}}$.

Observe that the type (ii) semigroups are precisely those 0-simple semigroups for which ker $P_{\{0\}}=E$. In other words, for any $a \in S \backslash E$, there exist $x, y \in S^{1}$ such that either $x a y \neq 0$ and $x a a^{-1} y=0$, or else $x a y=0$ and $x a a^{-1} y \neq 0$.

As in Section 5 , here too the simple semigroups having property $\mathbf{H}^{\mathrm{ker}}$ present a serious stumbling block. A discussion of this property in the context of Bruck extensions is contained in Section 8 .

We shall now have a closer look at the type (iii) semigroups. Recall that $S$ is categorical at zero if for any $a, b, c \in S, a b \neq 0$ and $b c \neq 0$ implies $a b c \neq 0$.

Proposition 6.5. Let $S$ be an inverse semigroup. Then $S$ is 0-simple, categorical at zero and kernel-harmonic if and only if $S$ is a combinatorial Brandt semigroup.

Proof. Direct part. By [4, Theorem 1.6], $S$ has a Brandt congruence $\theta$. Since $(S / \theta) / \mathscr{H}$ is combinatorial, $\rho=\theta^{\theta} \mathscr{H}$ is a combinatorial Brandt congruence, say $S / \rho \cong$ $B(1, I)$. Since $S$ is 0 -simple, $\{0\}$ is a class of $\rho$. We shall label the non-zero elements of $S$ with indices which indicate the image in $S / \rho$. By Theorem 6.4(iii), we have $\operatorname{ker} \rho=E$. Let the semilattices making up the kernel of $\rho$ be denoted by $Y_{i}$ for $i \in I$. Since $S$ is 0 -simple, for any $a_{i i}, b_{i i} \in Y_{i}$, there exist $x_{k l}, y_{p q} \in S$ such that $a_{i i}=x_{k l} b_{i i} y_{p q}$. Then $i=k=l=p=q$ so that $a_{i i}=x_{i i} b_{i i} y_{i i}$ where now $a_{i i}, x_{i i}, b_{i i}, y_{i i}$ are elements of the semilattice $Y_{i}$. It follows that $a_{i i} \leq b_{i i}$. By symmetry, we conclude that $a_{i i}=b_{i i}$. Therefore $|Y|=1$ for all $i \in I$, and so $\rho$ is an idempotent separating congruence. It follows that every nonzero idempotent of the 0 -simple inverse semigroup $S$ is primitive, whence $S$ is a Brandt semigroup. Then since $\mathscr{H}$ is a congruence, therefore idempotent pure, it follows that $S$ is combinatorial.

Converse. It is well known that $B(1, I)$ is congruence-free.

7. Trace-harmonic inverse semigroups. By analogy with the preceding section, we introduce the following condition pertaining to the trace.

DefinItIon 7.1. An inverse semigroup $S$ has property $\mathbf{H}^{\text {tr }}$ or is said to be trace-harmonic if for any congruences $\lambda$ and $\rho$ on $S, a \lambda=a \rho$ for some $a \in S$ implies that $\operatorname{tr} \lambda=\operatorname{tr} \rho$. 
Again an obvious modification of the proof of Lemma 5.2 gives

LEMMA 7.2. An inverse semigroup $S$ is trace-harmonic if and only if for every congruence $\rho$ on $S$ and every $a \in S$, we have $\operatorname{tr} \rho=\operatorname{tr} P_{a \rho}$.

Here too, we have a general classification theorem for trace-harmonic semigroups.

THEOREM 7.3. An inverse semigroup $S$ is trace-harmonic if and only if $S$ is of one of the following types:

(i) a simple trace-harmonic semigroup,

(ii) an ideal extension of a group $G$ by a 0-simple semigroup $T$ determined by $a$ surjective partial homomorphism $\varphi: T^{*} \rightarrow G$ and where every non-universal congruence on $T$ is idempotent separating.

Proof. Direct part. Let $S$ be a trace-harmonic semigroup and let $l$ be a proper ideal of $S$. For the Rees congruence $\rho_{I}$ and $a \in S \backslash I$, we then have $a \rho_{I}=a \varepsilon$ and thus $\operatorname{tr} \rho_{I}=\varepsilon$. It follows that $I$ has only one idempotent and is thus a group. Therefore $S$ has a unique ideal, which is a group, say $G$. As a consequence, we have that the Rees quotient $T=S / G$ is a 0 -simple semigroup. Since $G$ has an identity, say $e$, the extension $S$ is determined by the partial homomorphism $\varphi: T^{*} \rightarrow G$ given by $t \varphi=t e$. Then $\psi=\varphi \cup \iota_{G}$ is a retraction of $S$ onto $G$. If $\varphi$ does not map $T^{*}$ onto $G$, then there is an element $g \in G$ such that $\{g\}$ is a class of the congruence ${ }^{\psi} \varepsilon$ on $S$. But then $g^{\psi} \varepsilon=g \varepsilon$ so that, by $\mathbf{H}^{\text {tr }}$, we must have $\operatorname{tr}^{\psi} \varepsilon=\varepsilon$. But $\operatorname{tr}^{\psi} \varepsilon=\omega \neq \varepsilon$, a contradiction. Consequently $\varphi$ is surjective.

Now observe that $T$ is a homomorphic image of $S$ and $\mathbf{H}^{\text {tr }}$ is preserved under homomorphic image, whence $T$ is a 0 -simple trace-harmonic semigroup. But then every proper congruence on $T$ has equality trace, as required.

Converse. It suffices to consider a semigroup of type (ii). Let $\rho$ be a congruence on $S$.

Case 1. $\rho$ saturates $G$. Then $\left(\rho \vee \rho_{G}\right) / \rho_{G}$ is a proper congruence on $S / G \cong T$, hence idempotent separating by hypothesis. But $\rho_{G}$ is idempotent separating and thus $\rho \vee \rho_{G}$ is idempotent separating, whence $\rho$ is idempotent separating.

Case 2. $\rho$ does not saturate $G$. Hence there exist $a \in S \backslash G$ and $b \in G$ such that $a \rho b$. Thus $a a^{-1} \in e\left(\rho \vee \rho_{G}\right)$, whence $\left(\rho \vee \rho_{G}\right) / \rho_{G}$ is not idempotent separating. Consequently, we have $\rho \vee \rho_{G}=\omega$, from which it follows that every $\rho$ class meets $G$ and so $\rho$ is a group congruence.

Now if $\lambda$ and $\rho$ are congruences such that $\lambda$ saturates $G, \rho$ does not saturate $G$, and $a \lambda=a \rho$ for some $a \in S$, then by Corollary 3.11 it must be that $a \in G$. But the map $\psi=\varphi \cup \iota_{G}$ is a retraction of $S$ onto $G$ and by hypothesis there exists $b \in S \backslash G$ with $b \psi=a \psi$. Since ${ }^{\psi} \varepsilon=\sigma$, the least group congruence on $S$, we have $a \sigma b$ and thus $a \rho b$. But then $b \in a \rho=a \lambda \subseteq G$, a contradiction. Thus, if $a \lambda=a \rho$ for some congruences $\lambda$ and $\rho$ on $S$ and some element $a \in S$, then $\lambda$ and $\rho$ are either as in Case 1 , in which case $\operatorname{tr} \lambda=\operatorname{tr} \rho=\varepsilon$, or as in Case 2, in which case $\operatorname{tr} \lambda=\operatorname{tr} \rho=\omega$. Therefore $S$ has property $\mathbf{H}^{\operatorname{tr}}$.

Observe that 0 -simple inverse semigroups $S$ all of whose non-trivial congruences are idempotent separating are precisely those 0 -simple inverse semigroups in which $\operatorname{tr} P_{\{0\}}=$ $\varepsilon$. In other words, for any $e, f \in E_{S}, e \neq f$, there exist $x, y \in S^{1}$ such that either $x e y \neq 0$ and 
$x f y=0$ or else $x e y=0$ and $x f y \neq 0$. Further note that $\operatorname{tr} P_{\{0\}}=\varepsilon$ implies that $P_{\{0\}} \subseteq \mu$. Since $\mu \neq \omega$, it must be 0 -restricted, which by maximality of $P_{\{0\}}$ implies that $\mu \subseteq P_{\{0\}}$. Therefore $P_{\{0\}}=\mu$.

As in the preceding section, we can be more specific if we consider 0-simple inverse semigroups which are categorical at zero. In type (ii) semigroups in the above theorem this means that $G$ is a categorical ideal of $S$.

Proposition 7.4. Let $S$ be an inverse semigroup. Then $S$ is 0 -simple, categorical at zero and trace-harmonic if and only if $S$ is a Brandt semigroup.

Proof. Direct part. As in the proof of Proposition 6.5, $S$ has a combinatorial Brandt congruence $\rho$. Since $\rho \neq \omega$, we obtain by Theorem 7.3 that $\operatorname{tr} \rho=\varepsilon$ so that $\rho \subseteq \mu$. Thus $\mu$ induces a non-universal congruence $\bar{\mu}$ on $S / \rho$. But $S / \rho$ is congruence-free, which then yields that $\bar{\mu}=\varepsilon$ and so $\mu=\rho$. Consider distinct non-zero idempotents $e$ and $f$ of $S$. Then $\bar{e} \neq \bar{f}$ in $\bar{S}=S / \rho$, whence $\overline{e f}=0$ and so $e f=0$. Therefore $S$ is a 0 -simple inverse semigroup all of whose idempotents are primitive, so it must be a Brandt semigroup.

Converse. It is well-known that a Brandt semigroup is 0-simple, categorical at zero and has all non-universal congruences idempotent separating. By Theorem 7.3, it must be trace-harmonic.

8. Harmonic Bruck extensions. Our first objective in this section is to characterize the harmonic Bruck extensions of a given inverse monoid. This characterization is then used in order to obtain an example of a non-congruence-free, non-bisimple harmonic semigroup. Finally we construct an h-closed sd-class of inverse semigroups which is also closed under Bruck extensions.

Throughout this section, $T$ will denote an inverse monoid with identity 1 , semilattice of idempotents $E$ and group of units $G$. Moreover, we shall use $\alpha$ to denote a homomorphism of $T$ into $G$ and $S$ shall denote the corresponding Bruck extension $B(T, \alpha)$. We shall also reserve $\beta$ to denote the congruence on $S$ defined by $(m, g, n) \beta(p, h, q)$ if and only if $m=p$ and $n=q$ for any $(m, g, n),(p, h, q) \in S$.

It will be convenient to identify $T$ with the submonoid $\{0\} \times T \times\{0\}$ of $S$.

DEFINITION 8.1. (i) A congruence $\rho$ on $T$ shall be called $\alpha$-invariant, or simply an $\alpha$-congruence, if $\rho \subseteq{ }^{\alpha} \rho$.

(ii) For any proper ideal $I$ of $T$, an $\alpha$-congruence $\rho$ on $T$ shall be called a crossover $\alpha$-congruence with associated ideal $I$ if $\rho$ saturates $I$ and $\left.{ }^{\alpha} \rho\right|_{I}=\left.\rho\right|_{I}$.

Note that $I$ is uniquely determined by the conditions of Definition 8.1 (ii).

We shall make use of the description of congruences on $S$ as given in [8]. For convenience, we cite the relevant results here.

THEOREM 8.2 [8]. Every congruence on $S$ is an extension of a congruence on $T$. The congruences on $T$ which extend to $S$ are precisely the $\alpha$-congruences on $T$. Each $\alpha$-congruence $\rho$ on $T$ has exactly one extension $\hat{\rho}$ contained in $\beta$. For a non-group $\alpha$-congruence $\rho$ on $T$, there is at most one extension not contained in $\beta$, and this extension 
$\tilde{\rho}$ exists if and only if $\rho$ is a crossover $\alpha$-congruence. The non-group congruences on $S$ consist of all extensions of the non-group $\alpha$-congruences on $T$ together with the extensions $\hat{\rho}$ for $\rho$ a group $\alpha$-congruence on $T$.

For an $\alpha$-congruence $\rho$ on $T$, we have $(m, g, n) \hat{\rho}(p, h, g) \Leftrightarrow m=p, n=q$ and $g \rho h$. If moreover $\rho$ is a crossover $\alpha$-congruence with associated ideal $I$, then

$$
(m, g, n) \tilde{\rho}(p, h, q) \Leftrightarrow\left\{\begin{array}{l}
m=p, n=q, g \rho h, \quad \text { or } \\
m=p+1, n=q+1, h \in I,(h \alpha) \rho g, \quad \text { or } \\
p=m+1, q=n+1, g \in I,(g \alpha) \rho h .
\end{array}\right.
$$

We note that if $\rho$ is a crossover $\alpha$-congruence on $T$ with associated ideal $I$, then $I \cap G \rho=\varnothing$, whence $I \cap(I \alpha) \rho=\varnothing$.

Proposition 8.3 [8]. Let $\rho$ be an $\alpha$-congruence on $T$, and $(m, g, n) \in S$. Then

$$
(m, g, n) \hat{\rho}=\{m\} \times g \rho \times\{n\},
$$

while if $\rho$ is a crossover $\alpha$-congruence with associated ideal 1 , then $(m, g, n) \tilde{\rho}$ equals

$$
\begin{cases}(\{m\} \times g \rho \times\{n\}) \cup(\{m+1\} \times(g \alpha) \rho \times\{n+1\}) & \text { if } g \in I, \\ (\{m-1\} \times h \rho \times\{n-1\}) \cup(\{m\} \times g \rho \times\{n\}) & \text { if } g \rho(h \alpha) \text { for } h \alpha \in I \text { and } m n>0, \\ \{m\} \times g \rho \times\{n\} & \text { otherwise. }\end{cases}
$$

Corollary 8.4. If $\rho$ is a crossover $\alpha$-congruence on $T$, then

(i) $\operatorname{ker} \hat{\rho}=\operatorname{ker} \tilde{\rho}$, while $\operatorname{tr} \hat{\rho} \nsubseteq \operatorname{tr} \tilde{\rho}$,

(ii) $\hat{\rho}$ and $\tilde{\rho}$ have a congruence class in common.

Proof. (i) See [8].

(ii) By Proposition 8.3, we have $(0,1,0) \hat{\rho}=\{0\} \times 1 \rho \times\{0\}=(0,1,0) \tilde{\rho}$.

Proposition 8.5. If $T$ has a proper ideal $I$, then $\rho=\rho_{I} \cap{ }^{\alpha} \varepsilon$ is a crossover $\alpha$-congruence on $T$ with associated ideal $I$.

Proof. It is apparent that $\rho$ is an $\alpha$-congruence and that $\rho$ saturates $I$. It remains to show that $\left.\left.{ }^{\alpha} \rho\right|_{I} \subseteq \rho\right|_{I}$. Let $x, y \in I$ be such that $(x \alpha) \rho(y \alpha)$. Since $\rho \subseteq \rho_{I}$ and $I$ is a proper ideal of $T$, we have $G \cap I=\varnothing$ and $x \alpha, y \alpha \in G$ implies $x \alpha=y \alpha$. Thus $x \rho_{I} y$ and $x^{\alpha} \varepsilon y$, whence $x \rho y$, as required.

Corollary 8.6. If any Bruck extension of $T$ is trace-harmonic then $T$ is simple.

Proof. If $T$ is not simple, then by Proposition 8.5 there exists a crossover $\alpha$-congruence $\rho$ on $T$. By Corollary 8.4(ii), $\hat{\rho}$ and $\tilde{\rho}$ have a congruence class in common, and by Corollary 8.4(ii) we have $\operatorname{ker} \tilde{\rho}=\operatorname{ker} \hat{\rho}$ and $\operatorname{tr} \hat{\rho} \neq \operatorname{tr} \tilde{\rho}$. Thus $S$ does not have property $\mathbf{H}^{\text {tr }}$.

COROLlary 8.7. No simple non-bisimple $\omega$-semigroup is trace-harmonic. 
Proof. The simple $\omega$-semigroups are, up to isomorphism, Bruck extensions of finite chains of groups [6, XI.4]. Moreover, a Bruck extension of a monoid is bisimple if and only if the monoid is bisimple. But a finite chain of groups is bisimple if and only if it is simple. The result now follows from Corollary 8.6.

At this point, we offer an example which answers in the negative a question raised by Schein in [10]. We construct a simple inverse semigroup which has a congruence $\rho$ contained in $\mathscr{D}$, but $\rho$ is not determined by each of its classes. Let $S$ be the Bruck extension $B(T, \alpha)$ where $T$ is the two element chain $\{0,1\}$ with $0 \leq 1$, and $\alpha$ is the constant endomorphism of $T$ with image $\{1\}$. Then $\rho=\varepsilon$ is a crossover $\alpha$-congruence with associated ideal $\{0\}$. By Corollary 8.4(i) and (ii), respectively, $\hat{\rho} \neq \tilde{\rho}$ but they have a congruence class in common. Moreover, $\hat{\rho}=\varepsilon \subseteq \mathscr{D}$.

We return now to our investigations. Since our immediate goal is to characterize harmonic Bruck extensions, Corollary 8.6 directs our attention to Bruck extensions of simple inverse monoids. in $\beta$.

LEMMA 8.8. $T$ is simple if and only if each non-group congruence on $S$ is contained

Proof. It follows from Propositions 8.3 and 8.5 that if $T$ is not simple, then not every non-group congruence on $S$ is contained in $\beta$. Conversely, suppose that there exists a non-group congruence $\rho$ not contained in $\beta$. Let $\lambda=\left.\rho\right|_{T}$. Then by Theorem $8.2, \lambda$ must be a crossover $\alpha$-congruence on $T$ and $\tilde{\lambda}=\rho$. But then $T$ has a proper ideal, namely the ideal associated with $\lambda$. Thus $T$ is not simple.

We require one additional definition before proceeding to the characterization of harmonic Bruck extensions of a monoid $T$.

DEFinition 8.9. If $T$ is a monoid and $\alpha$ is a homomorphism from $T$ into its group of units, we say that $T$ is $\alpha$-harmonic if every $\alpha$-congruence on $T$ is determined by any one of its classes.

THEOREM 8.10. A Bruck extension $S=B(T, \alpha)$ is harmonic if and only $T$ is simple and $\alpha$-harmonic.

Proof. By Corollary 3.8, any two group congruences on $S$ with a congruence class in common must be equal. By Corollary 3.12, a group congruence and a non-group congruence on $S$ cannot have a congruence class in common. It follows that $S$ is harmonic if and only if every non-group congruence on $S$ is determined by any of its classes. By Corollary 8.6 , it is necessary that $T$ be simple if $S$ is to be harmonic. Assume that $T$ is simple. By Lemma 8.8, every non-group congruence on $S$ is contained in $\beta$ and so, by Theorem 8.2, must be of the form $\hat{\rho}$ for some $\alpha$-congruence $\rho$ on $T$. Now by Proposition 8.3, for $\alpha$-congruences $\lambda$ and $\rho$ on $T, \hat{\lambda}$ and $\hat{\rho}$ have a congruence class in common if and only if $\lambda$ and $\rho$ have a congruence class in common. Furthermore, by definition we have $\hat{\lambda}=\hat{\rho}$ if and only if $\lambda=\rho$. Thus $S$ is harmonic if and only if $T$ is simple and $\alpha$-harmonic.

COROLlaRy 8.11. If $T$ is simple and harmonic, then every Bruck extension of $T$ is harmonic. 
EXAMPLE 8.12. There exists a non-bisimple, non-congruence-free harmonic semigroup. For in [5, Theorem 2.12] Munn shows that for a particular semilattice $E$ constructed by C. J. Ash ([1], see also [5, Example 2.6]), the monoid $T_{E}^{*}$ is simple, non-bisimple and congruence-free. Since $T_{E}^{*}$ is not a group, the only homomorphism of $T_{E}^{*}$ into its group of units is the trivial one. The corresponding Bruck extension is a simple, non-bisimple harmonic monoid with non-trivial lattice of congruences.

Proposition 8.13. Let $T$ be simple.

(i) A homomorphic image of a Bruck extension of $T$ is either a group or else a Bruck extension of a homomorphic image of $T$.

(ii) If $T$ has the sd-property, then every Bruck extension of $T$ has the sd-property.

Proof. (i) Let $\rho$ be a non-group congruence on $S=B(T, \alpha)$ Since $T$ is simple, Lemma 8.8 yields $\rho \subseteq \beta$. Let $\rho_{0}=\left.\rho\right|_{T}$, so that by Theorem 8.2 we have $\rho=\hat{\rho}_{0}$. Now $\rho_{0}$ is $\alpha$-invariant and so $\alpha$ induces a homomorphism $\bar{\alpha}$ of $T / \rho_{0}$ into its group of units. We show that the map $\gamma: B(T, \alpha) \rightarrow B\left(T / \rho_{0}, \bar{\alpha}\right)$ given by $(m, g, n) \mapsto\left(m, g \rho_{0}, n\right)$ is a surjective homomorphism with induced congruence $\rho$, whence $B(T, \alpha) / \rho \cong B\left(T / \rho_{0}, \bar{\alpha}\right)$. That $\gamma$ is a homomorphism follows from the observation that by definition of $\bar{\alpha},(a \alpha) \rho=(a \rho) \bar{\alpha}$ for all $a \in T$. Since $\gamma$ is obviously surjective, it remains to verify that $\rho={ }^{\gamma} \varepsilon$. We have $(m, g, n) \gamma=(p, h, q) \gamma$ if and only if $m=p, n=q$ and $g \rho_{0} h$, which by definition means $(m, g, n) \hat{\rho}_{0}(p, h, q)$. Since $\rho=\hat{\rho}_{0}$, the result follows.

(ii) Suppose that $T$ has the sd-property. For any $v \in S=B(T, \alpha)$, the congruence $\rho=P_{(v)}$ saturates $\{v\}$. By Corollary 3.12, we see that $\rho$ is not a group congruence. Then by Lemma 8.8 and Theorem 8.2, we have $\rho=\hat{\rho}_{0}$ where $\rho_{0}=\left.\rho\right|_{T}$. Since $v \rho=\{v\}$, it follows from Proposition 8.3 that $\rho_{0}$ has a singleton class, say $g \rho_{0}=\{g\}$. But then $\rho_{0} \subseteq P_{\{g\}}$. Since $P_{\{g\}}=\varepsilon$ by hypothesis, we have $\rho_{0}=\varepsilon$ whence $P_{\{v\}}=\hat{\varepsilon}=\varepsilon$. Thus $S$ has the sd-property.

Definition 8.14. Let $\widetilde{\mho}$ be a class of inverse monoids. Then $\mathbf{B}(\widetilde{\Re})$ shall denote the class consisting of all Bruck extensions of members of $\mathfrak{F}$. Define $\mathbf{B}^{(i)}(\mathfrak{F})$ inductively as follows. Let $\mathbf{B}^{(0)}(\mathfrak{F})=\widetilde{F}$ and for each $i \geq 0, \quad \mathbf{B}^{(i+1)}(\mathfrak{F})=\mathbf{B}^{(i)}(\mathfrak{F}) \cup \mathbf{B}\left(\mathbf{B}^{(i)}(\mathfrak{F})\right)$. Finally, define $\mathbf{B}^{(\infty)}(\mathfrak{F})=\bigcup_{i=0}^{\infty} \mathbf{B}^{(i)}(\mathfrak{F})$.

CoRollary 8.15. Let $\mathscr{P}$ stand for either of the class properties $h$-closed or sd-class. If a class $\mathfrak{F}$ of inverse monoids has property $\mathscr{P}$, then so does $\mathbf{B}^{(\infty)}(\mathfrak{Y})$ and $\mathbf{B}^{(i)}(\mathscr{F})$ for each $i \geq 0$. Moreover, $\mathbf{B}^{(\infty)}(\mathfrak{F})$ is closed under the formation of Bruck extensions.

In particular, if we take $\mathfrak{F}$ to be the class consisting of all simple congruence-free inverse monoids together with all bisimple inverse monoids, then $\mathbf{B}^{(\infty)}(\mathfrak{F})$ is an h-closed sd-class closed under Bruck extensions and properly containing $\mathfrak{F}$. The union of $\mathbf{B}^{(\infty)}(\mathfrak{F})$ with the class $\mathscr{A}$ consisting of all inverse semigroups either congruence-free or bisimple is an h-closed sd-class which properly contains $\mathscr{A}$.

We conclude this section with a discussion, in the context of Bruck extensions, of the properties introduced in Sections 6 and 7. An argument virtually identical to the proof of Theorem 8.10 will establish that a Bruck extension $B(T, \alpha)$ has property $\mathbf{H}^{\mathrm{tr}}$ if and only if 
$T$ is simple and has property $\mathbf{H}^{\text {tr }}$ for $\alpha$-congruences. Furthermore, in view of the results of this section, it is straightforward to prove that a Bruck extension $B(T, \alpha)$ has property $\mathbf{H}^{\mathrm{ker}}$ if and only if $T$ has property $\mathbf{H}^{\mathrm{ker}}$ for $\alpha$-congruences.

9. Harmonic $E$-unitary inverse semigroups. We follow the approach taken by $\mathbf{N}$. Reilly [9] in his investigations of congruence-free inverse semigroups. We are able to present sufficient conditions on the parameters of the P-semigroup construction in order that a simple harmonic semigroup results. It is somewhat unfortunate that all known examples of P-semigroups satisfying these conditions are in fact bisimple and thus do not offer us additional examples of harmonicity. However, the potential exists.

We shall refer the reader to [9] for all terms used in this section which have not appeared elsewhere in the present paper.

Before we turn our attention to P-semigroups, we discuss the results of [9, Section 3] in the context of harmonicity. It was shown there that if $E$ is a dense tree without zero and $S$ is a full inverse subsemigroup of $T_{E}$ which is interval subtransitive on the Dedekind completion $\bar{E}$ of $E$, then the non-identity congruences on $S$ are group congruences. Of course, any two group congruences with a congruence class in common are equal. Furthermore, by Proposition 3.11, if a group congruence has a singleton class, then the $\mathscr{D}$-class of that element is a group ideal of $S$. But since interval subtransitivity implies subtransitivity of $S$, it follows that $S$ is simple. Thus no group congruence on $S$ can have a congruence class in common with $\varepsilon$ and so $S$ is harmonic. If $S \nsubseteq T_{E}^{*}$ then $S$ has non-trivial group congruences and is therefore not congruence-free. Moreover, if $S$ is not transitive on $E$, then $S$ is not bisimple.

We proceed now to consider P-semigroups. If $S$ is harmonic, then for a P-semigroup $P=P(G, X, Y)$ such that $S$ is isomorphic to $P / \gamma$ for some idempotent separating congruence $\gamma$ on $P$, it follows that $P / \mu$ is harmonic.

It would be useful if one could obtain necessary and sufficient conditions on the parameters $G, X$ and $Y$ in order that $P(G, X, Y) / \mu$, or even $P(G, X, Y)$ itself, should be harmonic. Once again, we should like to obtain simple, non-bisimple inverse semigroups in order to extend our collection of harmonic semigroups. Thus the action of $G$ on $X$ should be such that $G$ does not act transitively on $Y$, but does act subtransitively on $Y$, that is to say, for any $\alpha, \beta \in Y$ there exists $g \in G$ with $\alpha \geq g \beta$.

Proposition 9.1. Let $P=P(G, X, Y)$ be a P-semigroup for which $Y$ has no zero and $G$ is $Y$-primitive. Then $P / \mu$ is harmonic.

Proof. Since $G$ is $Y$-primitive if and only if the only normal congruences on the semilattice of idempotents of $P$ are $\varepsilon$ and $\omega$, we see that under the hypothesis, the non-identity congruences on $P / \mu$ are group congruences. Moreover, if $I$ is a proper ideal of $P$, then the Rees congruence modulo $I$ has equality trace and so $P / \mu$ has a zero. But $Y$ is isomorphic to $E_{P / \mu}$ and $Y$ has no zero by hypothesis. We see therefore that $P$ must be simple and so $P / \mu$ is simple. As before, Proposition 3.11 then implies that $P / \mu$ is harmonic. 
It was suggested in [9] that one should consider the works on 0-primitive groups of semilattice automorphisms to obtain examples of congruence-free inverse semigroups of the form $P / \mu$ where $P$ is simply the semidirect product of the semilattice and the 0 -primitive group. We could certainly take the same approach to try and obtain harmonic semigroups. Unfortunately, the customary practice when studying 0-primitive group actions is to consider transitive actions. All the references to 0-primitive groups cited in [9] deal only with transitive actions and the resulting semidirect products are therefore bisimple.

\section{REFERENCES}

1. C. J. Ash, Dense, uniform and densely subuniform chains, J. Austral. Math. Soc. Ser. A 23 (1977), 1-8.

2. T. E. Hall, Congruences and Green's relations on regular semigroups, Glasgow Math. J. 13 (1972), 167-175.

3. R. J. Koch, Comparison of congruences on regular semigroups, Semigroup Forum 26 (1983), 295-305.

4. G. Lallement and M. Petrich, Décompositions I-matricielles d'un demi-groupe, J. Math. Pures Appl. 45 (1966), 67-117.

5. W. D. Munn, Congruence-free inverse semigroups, Quart. J. Math. Oxford Ser. (2) 25 (1974), 463-484.

6. M. Petrich, Inverse Semigroups (Wiley-Interscience, 1984).

7. M. Petrich and S. A. Rankin, Congruences induced by transitive representations of inverse semigroups, Glasgow Math. J. 29 (1987), 21-40.

8. S. A. Rankin, Congruences on an inverse Bruck-Reilly semigroup: Part I. Non-group congruences, preprint.

9. N. R. Reilly, Congruence-free inverse semigroups, Proc. London Math. Soc. Ser. (3) 33 (1976), 497-514.

10. B. M. Schein, A remark concerning congruences on (0-)bisimple inverse semigroups, Semigroup Forum 3 (1971), 80-83.

11. G. I. Žitomirskiı̀, On imprimitivity relations of generalized groups of transformations, (in Russian), Mat. Sb. (N.S.) 73 (1967), 500-512.

\section{UNIVERSITY OF WESTERn ONTARIO \\ LONDON, CANADA}

